\title{
Stage-matched survival differences by ethnicity among gastric cancer patients of Asian ancestry treated in the United States
}

\author{
Ryan M. Rhome, MD, PhD ${ }^{1}$, Meng Ru, $\mathbf{M S}^{2}$, Erin Moshier, $\mathrm{MS}^{2}$, Madhu Mazumdar, $\mathrm{PhD}^{2}$, and \\ Michael H. Buckstein, MD, PhD ${ }^{3}$ \\ ${ }^{1}$ Department of Radiation Oncology, Indiana University, Indianapolis, IN \\ ${ }^{2}$ Department of Population Health Science and Policy, Icahn School of Medicine at Mount Sinai, \\ New York, NY \\ ${ }^{3}$ Department of Radiation Oncology, Icahn School of Medicine at Mount Sinai, New York, NY
}

\section{Abstract}

Background: Differences have been noted in overall survival (OS) in gastric cancer (GC) between trials conducted in Western versus Asian countries. The National Cancer Database (NCDB) reports outcomes and patient/disease variables relevant to OS.

\begin{abstract}
Methods: Using NCDB, we identified 89,558 adult patients with GC diagnosed 2004-2012 where self-reported race/ethnicity was available. Cox proportional hazard model was used to calculate multivariable hazard ratio (HR) of death, adjusting for race/ethnicity, age, gender, insurance, histology, grade, location, stage, and treatment type.
\end{abstract}

Results: After adjustment, Asian patients had improved OS (HR=0.74, CI 0.71-0.77). There were differences in OS between Asian ethnicities compared to White patients $(\mathrm{n}=69,945)$, notably with Korean ( $\mathrm{n}=1,249$, HR 0.70, CI 0.64-0.75), Chinese ( $\mathrm{n}=1,271$, HR 0.69, CI 0.64-0.75), and Indian/Pakistani patients ( $\mathrm{n}=492$, HR 0.68, CI 0.61-0.76). Japanese ( $\mathrm{n}=829$, HR 0.84, CI $0.77-$ 0.91 ) and Vietnamese ( $\mathrm{n}=560$, HR 0.79, CI 0.71-0.88) OS was also improved (p-values <0.0001), while Filipino patients showed no difference $(\mathrm{n}=415$, HR 1.00$)$. Black patients had slightly improved OS ( $\mathrm{n}=13,500$, HR 0.98, CI 0.95-1.00, p=0.035).

Conclusions: This analysis supports improved OS in Asian patients independent of stage, treatment, and known patient or disease characteristics in this large US cohort, and is the largest to define OS differences between Asian ethnicities.

\section{Keywords}

outcomes; disparities; national database

Corresponding Author: Michael Buckstein, M.D., Ph.D., Department of Radiation Oncology, Icahn School of Medicine at Mt. Sinai, 1184 5th Avenue, First Floor, New York, NY 10029, 212-241-7502. 


\section{Introduction:}

Gastric cancer (GC) affects approximately 990,000 new patients worldwide per year and is still a common cause of cancer-related death.[1] While oncologic surgical resection remains the standard of care for non-metastatic GC, optimal adjuvant therapies are debated. Postoperative chemoradiation[2], peri-operative chemotherapy[3], and other combinations [4] have been studied in prospective randomized trials. Interestingly, there were notable differences in overall survival (OS) when comparing these trials, with those conducted in predominantly Caucasian populations[2,3] showing worse stage-matched OS compared to those conducted in Asian populations.[4] Some single-institution reports prior to the publication of these trials showed mixed results on this matter, with some showing nonsignificant improvement on multivariable analysis[5] and others showing improved survival in smaller patient cohorts.[6-8] A large population registry analysis found no difference between Eastern and Western gastric cancer survival.[9] While it is possible that screening or surgical techniques might explain the differences, the possibility of inherent tumor biology differences[10] might also be a factor, calling into the question the generalizability of trial conducted with a single population.

The National Cancer Database (NCDB) reports population, treatment, and overall survival outcome data on a majority of cancer diagnoses in the United States. Within the racial designations captured, they provide more granular data regarding self-reported country of origin of Asian ancestry patients treated in the US. The influence of race in GC has been explored with older NCDB datasets.[11] Those authors showed that median survival for grouped Asian ancestry patients treated from 1995-1997 is improved after accounting for differences in confounders such as stage, treatment type, and age. However, these patients were all treated prior to the modern area where more recent trials have changed the standard of care. This has also been further supported by analyses of Surveillance, Epidemiology, and End Results (SEER)[12, 13] and smaller databases.[14, 15]

Here we sought use the epidemiological strengths of the NCDB on a modern cohort of patients using advanced statistical controls for confounding variables to further explore these findings within the racial designation "Asian" by separately analyzing major available ethnicity groups within this category.

\section{Methods:}

\section{Data source}

The NCDB is a project by the Commission on Cancer (COC) that amasses data on a majority (approximately 70\%) of the cancer diagnosed in the US each year. The source is available to investigators at $\mathrm{COC}$ centers and is de-identified. This project was determined to be Institutional Review Board exempt as all data was obtained through de-identified nationally available database.

\section{Patient selection}

Data was provided for patients diagnosed with GC between ages 18-90 diagnosed from 2004-2012, for a total of 101,426 patients. We included only patients with gastric 
adenocarcinoma, squamous cell carcinoma, or adenosquamous histology that had adequate information on race, stage, and follow-up or vital status, leaving a final cohort of $\mathrm{N}=89,558$.

\section{Variables and Endpoints}

The NCDB distinguishes race (coded as White, Black, Asian) from Hispanic ethnicity and these are individual variables both coded for each patient. We therefore focused our analysis on the race-based categories to ensure no duplication of patients, and we did not include Hispanic origin in our variable list. Asian patients were further characterized by selfreported ethnicity as coded by country of origin/ancestry. Our analysis of survival was limited to the most represented regions, which here were Chinese, Korean, Japanese, Vietnamese, Asian Indian/Pakistani, and Filipino. The remaining Asian patients were classified as "other" in our analyses but represent Hawaiian, Laotian, Thai, Kampuchean, Hmong, Polynesian, Samoan, New Guinean, and several others in the database. We additionally examined variables including age, gender, facility type, insurance type, year of diagnosis, Charlson-Deyo Comorbidity (CDC) Score, histology, American Joint Committee on Cancer (AJCC) clinical stage group, grade, gastric subsite location, and treatment type including surgery (gastrectomy vs ablation), radiation, and chemotherapy in all combinations. Insurance status was summarized as government insurance (Medicare, Medicaid, other), private insurance, or uninsured. Facility type was dichotomized as academic or non-academic. CDC Index values were truncated by NCDB into 0,1 and $\geq 2$.

The primary endpoint of the analysis was OS, defined as the time from diagnosis to death or last follow-up. We additionally examined factors that influence OS in the entire cohort and specifically the Asian subgroup.

\section{Statistical analysis}

Patient, facility, and disease-related baseline characteristics were summarized within each racial group. Continuous variables were reported as median (range) and nominal variables were reported as $\mathrm{N}(\%)$. The associations between racial groups (Asian vs. non-Asians) and each of the prognostic factors were evaluated by adjusted odds ratios (OR) and $95 \%$ confidence intervals (CI) estimated from a multivariable logistic regression model, adjusting for all variables outlined above.

OS was estimated using the method of Kaplan-Meier with comparisons among racial groups made using log-rank tests. Analyses were stratified by stage due to heterogeneity with respect to survival and treatment options. OS was further compared within different Asian ethnicities with white patients as the reference. Cox proportional hazards $(\mathrm{CPH})$ models were used to estimate multivariable Hazard Ratios (MHRs) for all variables outlined above and corresponding 95\% CIs on both the entire cohort and Asian cohort. All models also include tests for interaction between stage and treatment, assessed for clinical and statistical significance.

All statistical analyses described above were performed using SAS Version 9.4 (SAS Institute, Cary, NC). Hypothesis testing was two-sided and conducted at the 5\% level of significance. 


\section{Missing Data}

Approximately $3 \%$ of facility type and insurance type were missing and handled by multiple imputation using chained equations. Using the IVEware software system on the SAS platform, we initiated a sequential regression imputation by building models for each variable with missing data, to be imputed based on a univariable logistic, ordinal, multinomial logistic, or predictive mean matching regression conditional on all other variables.[16] We performed 10 repetitions of this procedure to generate 50 imputed datasets for stable estimates, which were later combined to summarize the effect estimates using Rubin's rules executed by MIANALYZE procedure in SAS in subsequent analysis. This method shows advantages to alternatives such as complete case analysis with respect to analytic bias when the assumption that data is missing at random holds.[17]

\section{Results:}

\section{Baseline characteristics}

The baseline characteristics of each cohort are summarized in Table 1. Notably, Asian patients were more likely to be under 60 years old, be treated at an academic facility, have non-government insurance, have lower co-morbidity score, present at earlier stage, and were less likely to have lesions in the gastric cardia. In this cohort, 27.7\% of Asian patients were diagnosed at Stage I compared to $22.4 \%$ of White patients. Clinically, Asian ancestry patients were more likely to have gastrectomy and radiation but not chemotherapy. These differences were then taken into account in multivariable Cox regression model of OS.

\section{Factors predicting OS}

In Table 2 we report the variables that predict for OS in the entire cohort and the Asian subgroup specifically. The hazard ratios (HR) reported are adjusted in the multivariable model for all other variables reported to account for imbalances noted in the baseline characteristics above. As an overall cohort, Asian patients have better OS than white patients when controlling for confounding baseline characteristics and treatment modalities (HR $0.74,9 \% \%$ CI $0.71-0.77, \mathrm{p}<0.0001$ ). Figure 1 A-D shows the survival curves for White, Black, and Asian race groups stratified by stage (combined stages in Supplemental Figure 1). Though all stages showed statistically significantly higher OS in Asian patients $(\mathrm{p}<0.0001)$, the magnitude of this difference diminishes with increasing stage. Stage IV patients had a small but statistically significant difference in OS. A smaller difference is observed between Black and White patients.

\section{Effect of ethnicity on OS}

Figure 2 shows the survival curves for each Asian ethnicity, divided by self-reported region of ancestry, along with the White patients for comparison. Korean patients visually appear to have higher OS, followed by Chinese patients. Figure 3 A-D further separates these curves by stage with significant differences by log rank $(\mathrm{p}<0.0001)$. Survival differences were again detected even in Stage IV disease.

The adjusted HR of these differences are illustrated in a forest plot in Figure 4 with HR of death and 95\% CI for each of the most represented ethnicities compared to White patients. 
Most Asian ethnicities, with the exception of Filipinos (HR 1.00), had a significantly better survival after adjusting for all known confounders with HR ranging from 0.68 (Indian/ Pakistani) to 0.84 (Japanese), all $\mathrm{p}<0.0001$. Black patients had a borderline improved OS (HR 0.98, 95\% CI 0.95-1.00, $\mathrm{p}=0.035$ ).

\section{Discussion:}

Despite the caveats that exist in comparing outcomes between large studies, there is improved OS survival of GC patients treated in large randomized trials conducted in Asia compared to Western cohorts.[2-4] This difference in outcomes has also been suggested by single institution experiences[8], smaller databases[14, 15], and earlier analyses of large databases such as SEER $[12,13]$, though other studies have not shown differences.[9] Screening practices[18, 19], variation in regional treatment paradigms [20], environmental exposures, stage migration[21], and underlying biological variations[22-24] including tumor immunity signatures[10] have been hypothesized as contributors to observed differences. Characterizing these outcomes in different populations is important given implications to personalized medicine and interpreting previous practice-defining randomized controlled trials. For instance, debates on the extent of lymph node dissection[20, 25-27] are driven largely by discrepancies in regionally-conducted trials.

We sought to use the NCDB as a source of patients treated in the US to determine if survival differences exist outside of those explainable by healthcare practice differences or stagedependent diagnoses. NCDB has been used to broadly describe the influence of Asian race on outcomes in GC using patients with survival data available only on 1995-1997[11], prior to the publication of all modern practice-defining studies.[2-4] Treatment type is likely to influence outcomes, but is not independent from stage. Therefore this is a difficult variable to include in any model without accounting for that influence. In addition to updating this with a modern cohort and larger patient numbers, we set an interaction variable between treatment type (in all combinations) and upfront clinical stage, as it would be expected that the contribution of any specific combination of treatments would be stage-dependent. Even including this interaction, Asian race overall remained a significant prognostic factor for survival (Table 2). We note here that this interaction was not analyzed to determine the individual impact of stage-specific treatments in GC, as this would require further analysis such as propensity matching which was outside the scope of this analysis and would be difficult to interpret given the limitations of certain variables such as chemotherapy type or cycles. We do however feel that for the purpose of this study, namely to account for clinical differences between groups, this interaction is representative and thorough. Interestingly, when we added location in the stomach, we did not see associations between OS and subsite in the group as a whole or the Asian subset. The latter was more likely to have cancer in the antrum, but this did not affect outcomes after accounting for other confounders in the model, suggesting that location does not independently predict for outcomes in this cohort.

In contrast, when comparing patients listed as Black race to White patients, the effective difference is small though it achieved significance due to large patient numbers. When looking at the entire cohort, different factors influenced the overall GC population compared with the Asian population alone (Table 2). Given such a large dataset, it is not surprising that 
many clinical and pathologic factors are associated with survival, though the significant predictors found are generally expected to predict for survival (such as tumor grade, stage, co-morbidity score). Many show common and analogous HR for survival in both the entire and the Asian cohort. For example, treatment at academic facilities was associated with improved HR of death in the total group (0.84) and the Asian subset (0.83), indicating that after adjustment for differences in rate of treatment at these facilities, the improved HR remains similar for both.

We also sought to give additional insight into the contribution of ethnicity to survival differences seen in Asian patients. The definition of race versus ethnicity is variable and can depend on the context in which it is being studied. Overall, the designation of ethnicity includes additional factors such as geography and culture beyond biological traits such as skin color. The NCDB classifies Asian patients under the race variable with further delineation of their ethnicity based on the self-reported country/region of origin. Further obscuring this point is that NCDB does have a separate variable for Hispanic ethnicity. The broad grouping of patients as "Asian" is a useful but blunt tool for these hypothesisgenerating discussions, and additional information that further dissects this would be useful to determination of mechanistic and possibly targetable causes of these differences.

OS differences were noted between ethnicities within Asian patients in our analysis after controlling for known confounders (Figure 4). This difference persisted when divided by stage (Figure 3), though magnitude was more evident in earlier stages. This is, to our knowledge, the largest report of its kind to describe these specific differences as associated with survival after controlling for confounders. We chose to use upfront AJCC clinical stage here because it more accurately reflects the stage at presentation, and because many patients (ex. metastatic) would not receive surgery and therefore not have a pathologic stage. Indian/ Pakistani, Chinese, and Korean patients had the best HR for survival, with Filipino patients showing no differences in outcome compared to White patients, which corroborates previous smaller local database results.[6,14] Specifically, the improved survival seen in Korean patients (Figure 2-3) provides additional context to the trial conducted in Korean patients which illustrated the hypothesized ethnicity-based survival discrepancy in GC when compared to older trials conducted in Western countries.[4] Possible genetic variations in expression enriched in certain populations [28, 29] or even geographic differences in $H$. pylori gene expression may contribute to this finding.[30, 31] Aside from survival, regional differences have also been noted in response to treatment.[32]

The implications of this analysis extend beyond interpreting OS between studies conducted in Asian versus non-Asian populations. The efficacy of specific interventions should be considered in light of these data. In addition to adjuvant therapies[2-4], the discordant data on effect of surgical techniques such as extent of lymph node dissection[25-27] may be at least partially explained by biological underpinnings rather than hypothesized surgical experience.[20] With multiple options available in management of GC, it may be appropriate to investigate tailoring data-driven approaches to patients based on the context in which they were studied. 
This study has several limitations. This database captures an incomplete majority of patients treated in the US and is subject to the limitations of any retrospective dataset. Furthermore we are only able to report on the individual variables provided. Despite our best statistical efforts to control, unknown or unmeasured cofounders may exist that could change our conclusions. For example, the incidence of diffuse versus intestinal subtype is incompletely coded in the database and these have known differences in disease behavior. Signet ring type variants are also incompletely coded, though the contribution to outcome is debated.[33] Tumor grade was well captured in this dataset further minimizing the potential impact of histological subset. The racial designations reported herein are patient-reported and therefore are subject to some variation. NCDB does not differentiate between patients born in the US versus those that immigrated or came for treatment, and some SEER studies have shown possible differences within these populations.[34] Furthermore, Hispanic ethnicity is coded as a separate, unique designation from race in this dataset. We did not separately evaluate the patients of Hispanic ethnicity, which in other single institution and databases studies has not shown to affect survival in GC.[7, 15] Because of these factors and the absence of more nuanced biological information such as tumor genetics, we have limited the scope of our conclusions to hypothesis-generating differences in outcomes that are not explained by known clinical factors. Mechanistic underpinnings of these differences would require detailed analysis of tumors from each of these groups to determine a clear biologic determinant of outcome.

Here we report that, in a modern large-scale dataset, GC patients of Asian descent treated in the US have improved survival when adjusting for known confounders. Furthermore, subdividing this group into self-reported ethnicities yields additional differences with some having numerically better (Korean, Indian/Pakistani, Chinese) or worse (Japanese) survival compared to the broader Asian cohort, and others having equivalent survival to non-Asian groups studied (Filipino). Since all these patients were treated in the US and there are differences between Asian subgroups, this data highly suggests underlying biological differences driving the survival differences. Factors that positively influenced survival in Asian population alone included male gender, while insurance status, histology, and gastric subsite did not affect their survival as they did in the full cohort. In addition to informing discussions of prognosis in specific groups, these data should serve as a hypothesisgenerating report to further investigate the mechanism of these differences and should be considered when attempting to extrapolate results of clinical trials between ethnic populations.

\section{Supplementary Material}

Refer to Web version on PubMed Central for supplementary material.

\section{Acknowledgements:}

The authors wish to acknowledge the support of the Biostatistics Shared Resource Facility, Icahn School of Medicine at Mount Sinai, and NCI Cancer Center Support Grant P30 CA196521-01. 


\section{References:}

1. Karimi P, et al., Gastric cancer: descriptive epidemiology, risk factors, screening, and prevention. Cancer Epidemiol Biomarkers Prev, 2014 23(5): p. 700-13. [PubMed: 24618998]

2. Macdonald JS, et al., Chemoradiotherapy after surgery compared with surgery alone for adenocarcinoma of the stomach or gastroesophageal junction. N Engl J Med, 2001 345(10): p. $725-$ 30. [PubMed: 11547741]

3. Cunningham D, et al., Perioperative chemotherapy versus surgery alone for resectable gastroesophageal cancer. N Engl J Med, 2006 355(1): p. 11-20. [PubMed: 16822992]

4. Lee J, et al., Phase III trial comparing capecitabine plus cisplatin versus capecitabine plus cisplatin with concurrent capecitabine radiotherapy in completely resected gastric cancer with D2 lymph node dissection: the ARTIST trial. J Clin Oncol, 2012 30(3): p. 268-73. [PubMed: 22184384]

5. Gill AJ and Martin IG, Survival from upper gastrointestinal cancer in New Zealand: The effect of distance from a major hospital, socio-economic status, ethnicity, age and gender. ANZ J Surg, 2002 72(9): p. 643-6. [PubMed: 12269915]

6. Theuer CP, Asian gastric cancer patients at a southern California comprehensive cancer center are diagnosed with less advanced disease and have superior stage-stratified survival. Am Surg, 2000 66(9): p. 821-6. [PubMed: 10993608]

7. Yao JC, et al., Clinicopathologic behavior of gastric adenocarcinoma in Hispanic patients: analysis of a single institution's experience over 15 years. J Clin Oncol, 2005 23(13): p. 3094-103. [PubMed: 15860869]

8. Chen Y, et al., Asian gastric cancer patients show superior survival: the experiences of a single Australian center. Gastric Cancer, 2015 18(2): p. 256-61. [PubMed: 24858392]

9. Gill S, et al., Asian ethnicity-related differences in gastric cancer presentation and outcome among patients treated at a canadian cancer center. J Clin Oncol, 2003 21(11): p. 2070-6. [PubMed: 12775731]

10. Lin SJ, et al., Signatures of tumour immunity distinguish Asian and non-Asian gastric adenocarcinomas. Gut, 2015 64(11): p. 1721-31. [PubMed: 25385008]

11. Al-Refaie WB, et al., The impact of ethnicity on the presentation and prognosis of patients with gastric adenocarcinoma. Results from the National Cancer Data Base. Cancer, 2008 113(3): p. 461-9. [PubMed: 18553367]

12. Wang SJ, et al., Conditional survival in gastric cancer: a SEER database analysis. Gastric Cancer, 2007 10(3): p. 153-8. [PubMed: 17922092]

13. Wang J, Sun Y, and Bertagnolli MM, Comparison of gastric cancer survival between Caucasian and Asian patients treated in the United States: results from the Surveillance Epidemiology and End Results (SEER) database. Ann Surg Oncol, 2015 22(9): p. 2965-71. [PubMed: 25631065]

14. Kim J, et al., Disparities in gastric cancer outcomes among Asian ethnicities in the USA. Ann Surg Oncol, 2009 16(9): p. 2433-41. [PubMed: 19582508]

15. Kim J, et al., Race and ethnicity correlate with survival in patients with gastric adenocarcinoma. Ann Oncol, 2010 21(1): p. 152-60. [PubMed: 19622590]

16. White IR, Royston P, and Wood AM, Multiple imputation using chained equations: Issues and guidance for practice. Stat Med, 2011 30(4): p. 377-99. [PubMed: 21225900]

17. White IR and Carlin JB, Bias and efficiency of multiple imputation compared with complete-case analysis for missing covariate values. Stat Med, 2010 29(28): p. 2920-31. [PubMed: 20842622]

18. Taylor VM, et al., Gastric cancer in asian american populations: a neglected health disparity. Asian Pac J Cancer Prev, 2014 15(24): p. 10565-71. [PubMed: 25605140]

19. Hamashima C, Current issues and future perspectives of gastric cancer screening. World $\mathrm{J}$ Gastroenterol, 2014 20(38): p. 13767-74. [PubMed: 25320514]

20. Noguchi Y, et al., Is gastric carcinoma different between Japan and the United States? Cancer, 2000 89(11): p. 2237-46. [PubMed: 11147594]

21. Bunt AM, et al., Surgical/pathologic-stage migration confounds comparisons of gastric cancer survival rates between Japan and Western countries. J Clin Oncol, 1995 13(1): p. 19-25. [PubMed: 7799019] 
22. Lei YY, et al., The clinicopathological parameters and prognostic significance of HER2 expression in gastric cancer patients: a meta-analysis of literature. World J Surg Oncol, 2017 15(1): p. 68. [PubMed: 28327158]

23. Long ZW, et al., Association of IL-17 polymorphisms with gastric cancer risk in Asian populations. World J Gastroenterol, 2015 21(18): p. 5707-18. [PubMed: 25987798]

24. Xue H, et al., The effect of XPD/ERCC2 polymorphisms on gastric cancer risk among different ethnicities: a systematic review and meta-analysis. PLoS One, 2012 7(9): p. e43431. [PubMed: 23028453]

25. Kim S, et al., An observational study suggesting clinical benefit for adjuvant postoperative chemoradiation in a population of over 500 cases after gastric resection with D2 nodal dissection for adenocarcinoma of the stomach. Int J Radiat Oncol Biol Phys, 2005 63(5): p. 1279-85. [PubMed: 16099596]

26. Cuschieri A, et al., Patient survival after D1 and D2 resections for gastric cancer: long-term results of the MRC randomized surgical trial. Surgical Co-operative Group. Br J Cancer, 1999 79(9-10): p. 1522-30. [PubMed: 10188901]

27. Bonenkamp JJ, et al., Extended lymph-node dissection for gastric cancer. N Engl J Med, 1999 340(12): p. 908-14. [PubMed: 10089184]

28. $\mathrm{Hu} \mathrm{N}$, et al., Genome-wide association study of gastric adenocarcinoma in Asia: a comparison of associations between cardia and non-cardia tumours. Gut, 2016 65(10): p. 1611-8. [PubMed: 26129866]

29. Li Z, et al., IL-17A and IL-17F polymorphisms and gastric cancer risk: a meta-analysis. Genet Mol Res, 2015 14(2): p. 7008-17. [PubMed: 26125910]

30. Miura M, et al., Differential oncogenic potential of geographically distinct Helicobacter pylori CagA isoforms in mice. Int J Cancer, 2009 125(11): p. 2497-504. [PubMed: 19588494]

31. Ang TL, et al., Racial differences in Helicobacter pylori, serum pepsinogen and gastric cancer incidence in an urban Asian population. J Gastroenterol Hepatol, 2005 20(10): p. 1603-9. [PubMed: 16174081]

32. Satoh T, et al., Lapatinib plus paclitaxel versus paclitaxel alone in the second-line treatment of HER2-amplified advanced gastric cancer in Asian populations: TyTAN--a randomized, phase III study. J Clin Oncol, 2014 32(19): p. 2039-49. [PubMed: 24868024]

33. Taghavi S, et al., Prognostic significance of signet ring gastric cancer. J Clin Oncol, 2012 30(28): p. 3493-8. [PubMed: 22927530]

34. Kirchoff DD, et al., Overall Survival Is Impacted by Birthplace and Not Extent of Surgery in Asian Americans with Resectable Gastric Cancer. J Gastrointest Surg, 2015 19(11): p. 1966-73.

[PubMed: 26307345] 


\section{Synopsis for Table of Contents:}

Asian patients with gastric cancer in the United States have improved survival compared to White counterparts. Differences in survival exist between Asian ethnicities. 

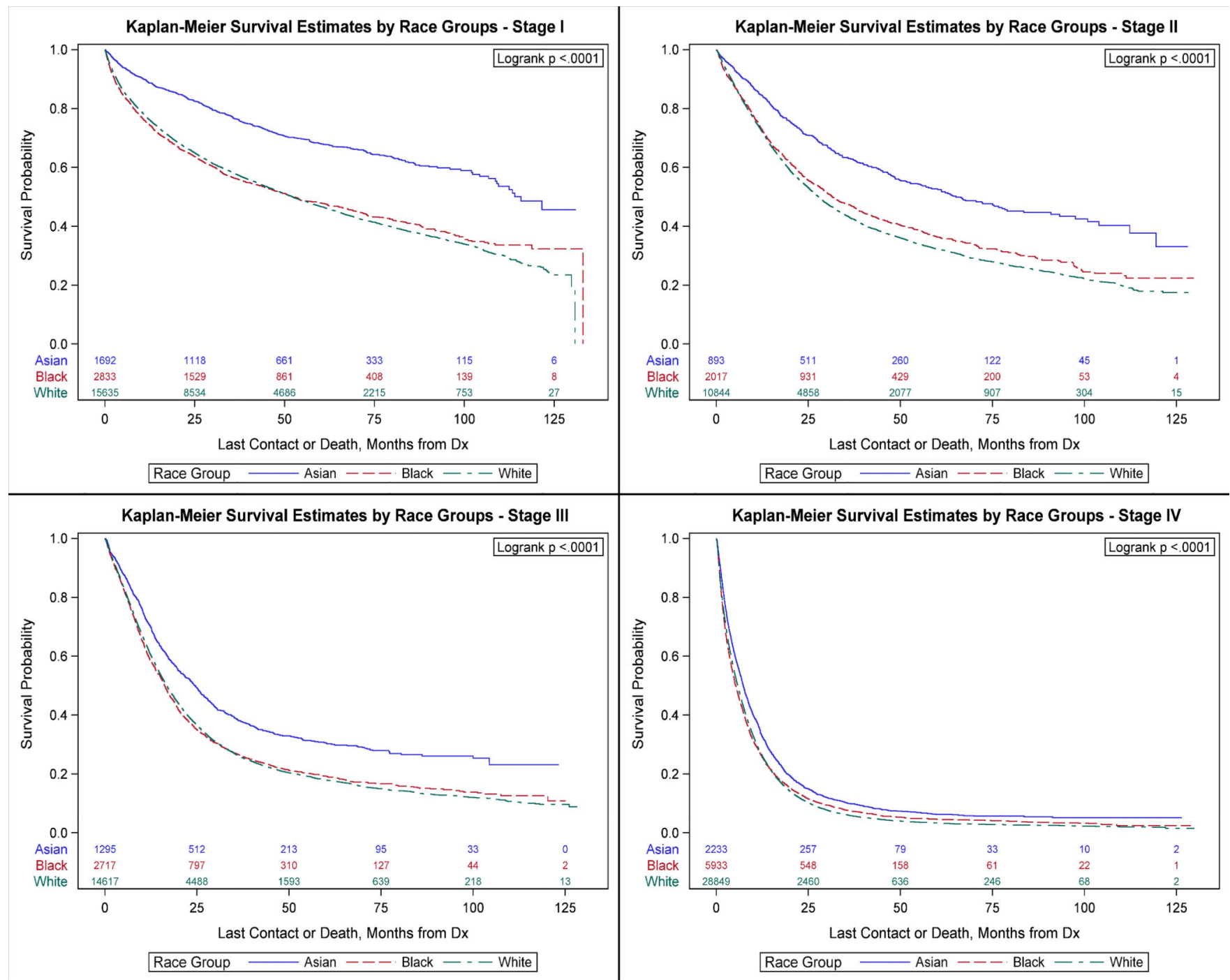

Figure 1A-D: Survival estimates by overall race group and stage.

Stage I (A), II (B), III (C), and IV (D).

Abbreviation: Dx: diagnosis 


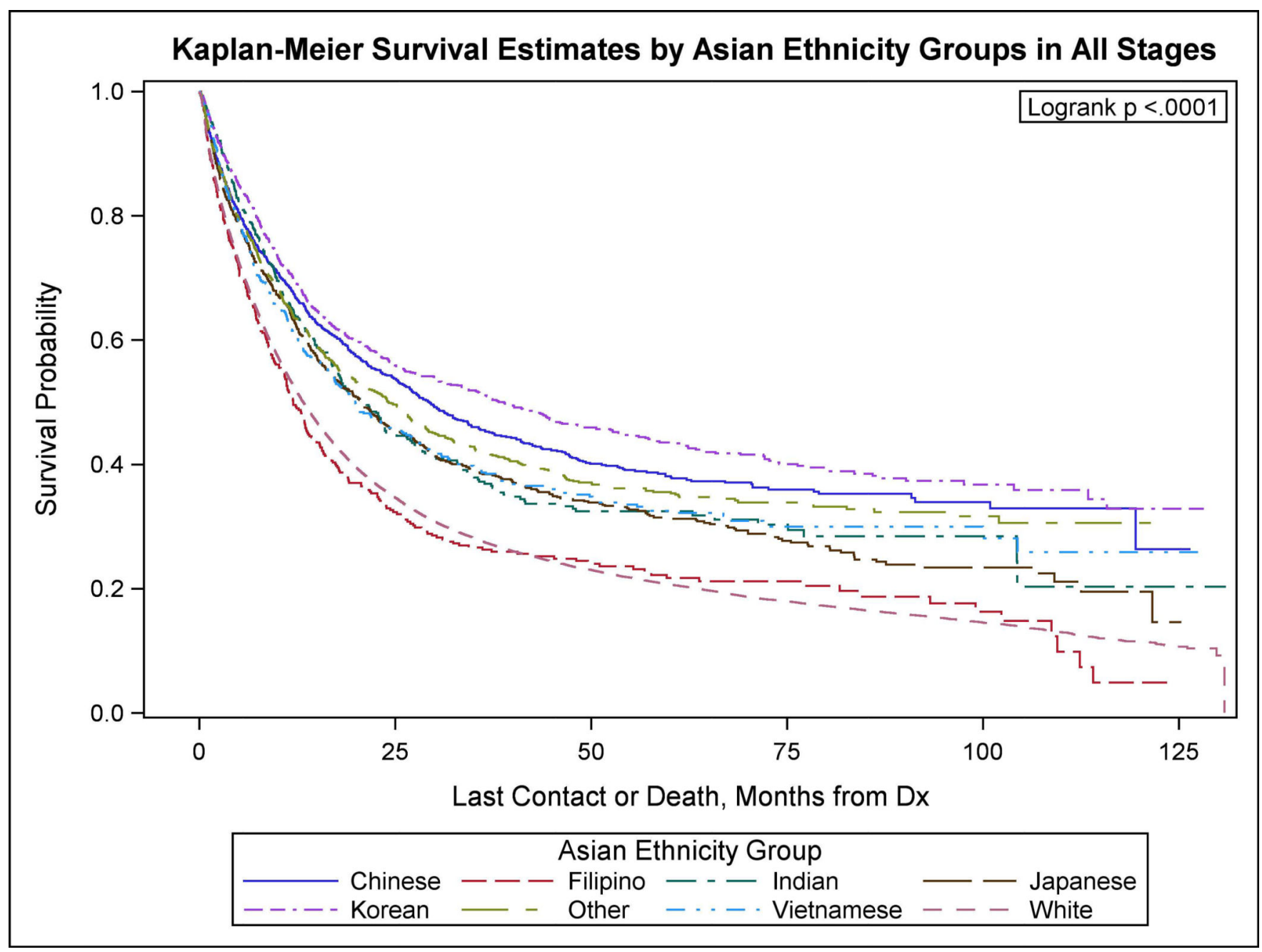

Figure 2: Survival estimates by Asian ethnicity subgroup compared to white patients, all stages. Abbreviation: Dx: diagnosis 

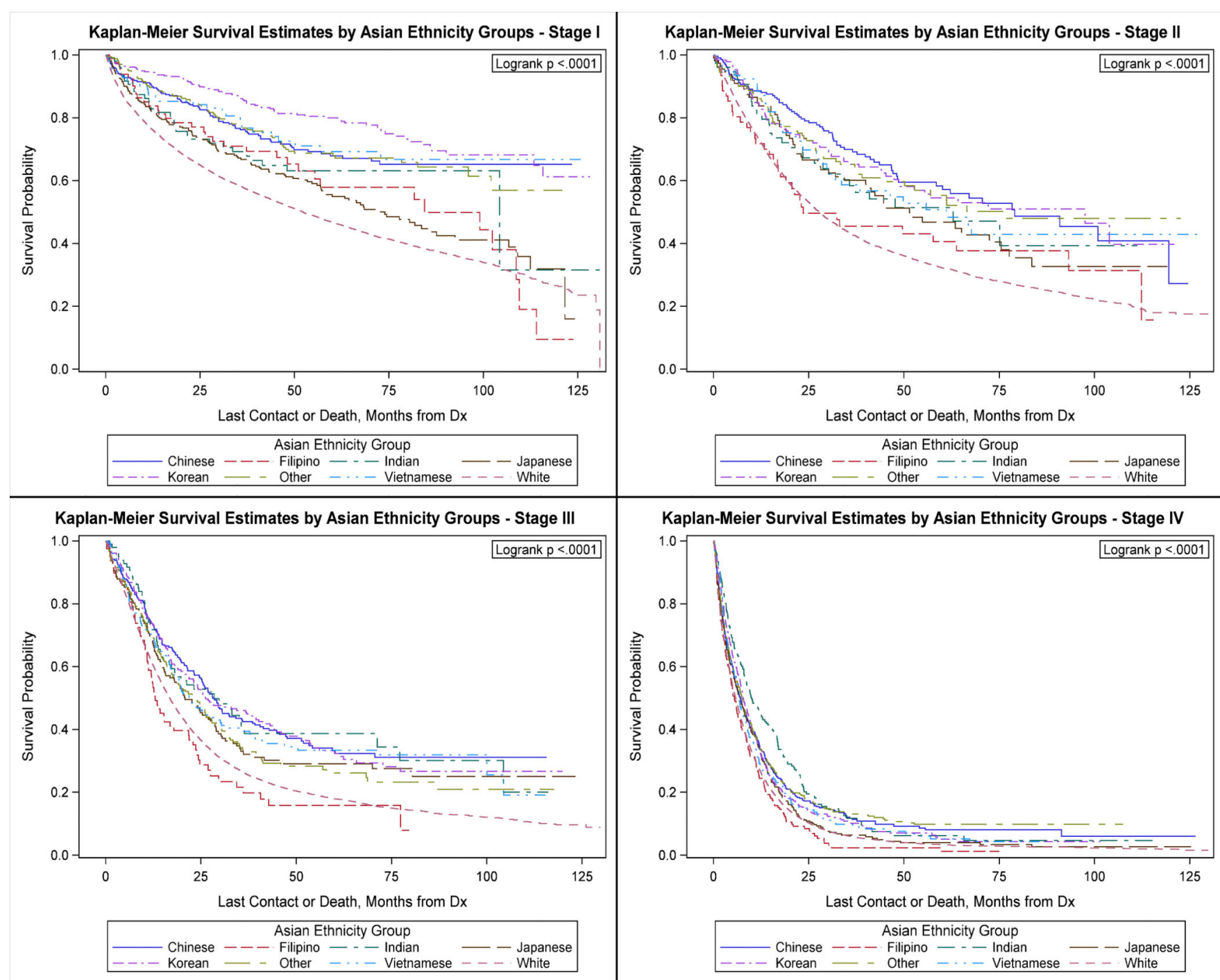

Figure 3A-D: Survival estimates by Asian ethnicity subgroup and stage. Stage I (A), II (B), III (C), and IV (D).

Abbreviation: Dx: diagnosis 


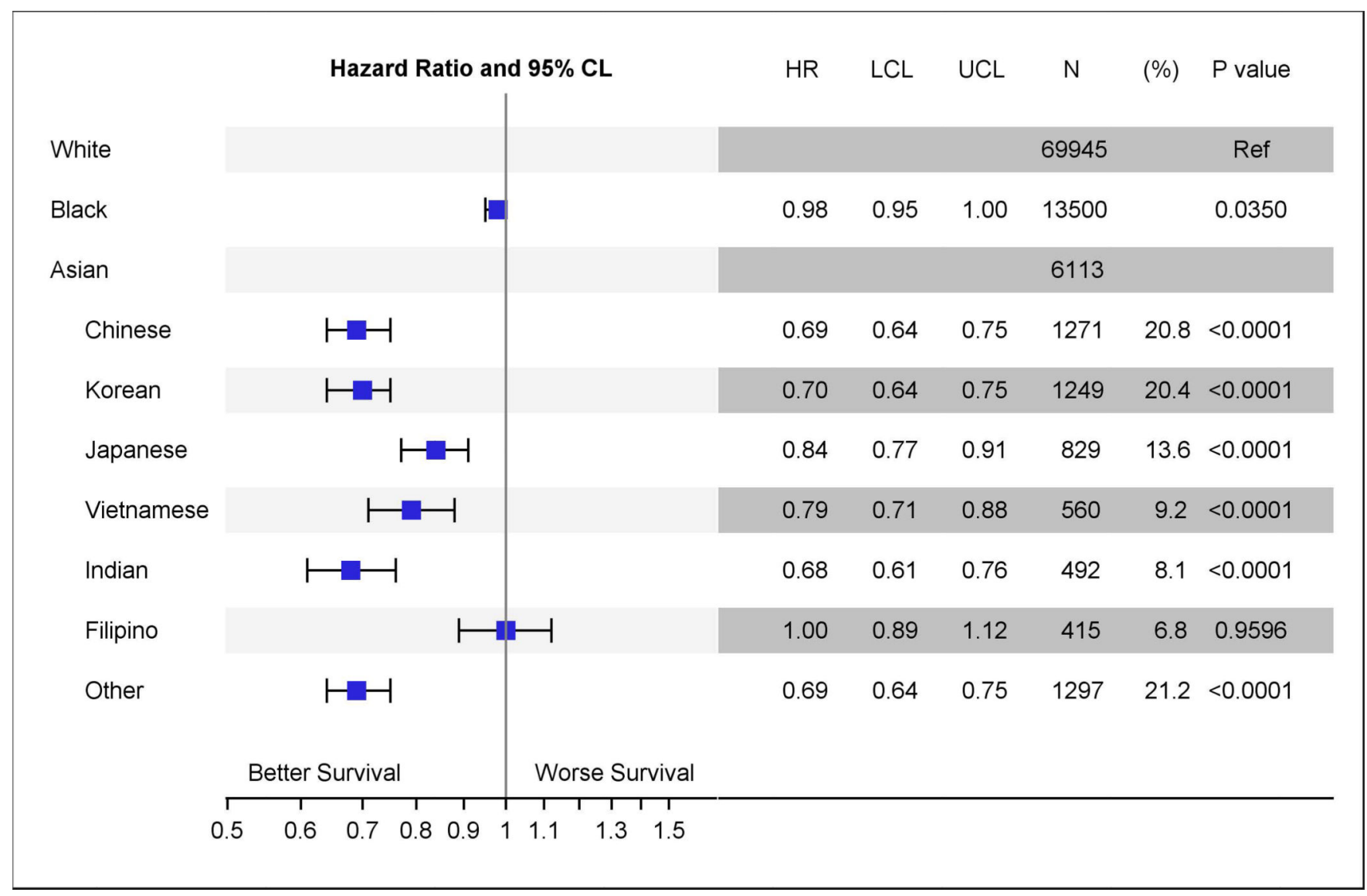

Figure 4: Hazard ratios for Overall Survival by ethnicity.

Abbreviation: HR: hazard ratio; CL: confidence limit; LCL: lower confidence limit; UCL: upper confidence limit; ref: reference 
Table 1.

Baseline characteristics by race group.

$\mathrm{N}=89,558$. Multivariable OR was estimated from the full logistic model including all covariates presented in this table comparing Asian to White and Black as others.

\begin{tabular}{|c|c|c|c|c|c|c|}
\hline $\mathbf{n}$ & & $\begin{array}{l}\text { White } \\
69945\end{array}$ & $\frac{\text { Black }}{13500}$ & $\frac{\text { Asian }}{6113}$ & OR $(95 \% \mathrm{CI})$ & $\mathrm{p}$ \\
\hline \multirow[t]{2}{*}{ Age> 60 n (\%) } & No & 20912 ( 29.9) & $5027(37.2)$ & $2229(36.5)$ & Ref & \\
\hline & Yes & $49033(70.1)$ & 8473 ( 62.8) & 3884 ( 63.5) & $0.92(0.89,0.95)$ & $<.0001$ \\
\hline Gender n (\%) & Male & 46770 ( 66.9) & 7963 ( 59.0) & $3548(58.0)$ & $0.98(0.95,1.01)$ & 0.1637 \\
\hline \multirow[t]{2}{*}{ Facility Type n(\%) } & Others & $38276(54.7)$ & $6270(46.4)$ & 3015 ( 49.3) & Ref & \\
\hline & Academic & 31699 ( 45.3) & $7230(53.6)$ & 3098 ( 50.7) & $1.09(1.06,1.12)$ & $<.0001$ \\
\hline Insurance Type n (\%) & Government & 44181 ( 63.2) & 8588 ( 63.6) & $3545(58.0)$ & $0.84(0.80,0.88)$ & $<.0001$ \\
\hline \multirow[t]{3}{*}{ CDC Score n (\%) } & 0 & $49022(70.1)$ & $8822(65.3)$ & $4632(75.8)$ & Ref & \\
\hline & 1 & $15279(21.8)$ & $3273(24.2)$ & $1163(19.0)$ & $1.03(0.97,1.09)$ & 0.3691 \\
\hline & 2 & $5644(8.1)$ & 1405 ( 10.4$)$ & $318(5.2)$ & $0.72(0.66,0.78)$ & $<.0001$ \\
\hline \multirow[t]{2}{*}{ Histology n (\%) } & Adeno & 69014 ( 98.7) & 13257 ( 98.2) & $6079(99.4)$ & Ref & \\
\hline & $\mathrm{SCC}$ & $259(0.4)$ & $43(0.3)$ & $12(0.2)$ & $0.96(0.63,1.45)$ & 0.8450 \\
\hline \multirow{2}{*}{ AJCC Stage n (\%) } & 3 & 14617 ( 20.9) & $2717(20.1)$ & $1295(21.2)$ & $0.94(0.90,0.99)$ & 0.0281 \\
\hline & 4 & $28849(41.2)$ & 5933 ( 43.9) & $2233(36.5)$ & $0.97(0.92,1.03)$ & 0.3149 \\
\hline \multirow[t]{4}{*}{ Grade n (\%) } & Well Diff & 13207 ( 18.9) & $2493(18.5)$ & 959 ( 15.7$)$ & Ref & \\
\hline & Mod Diff & $17102(24.5)$ & $3384(25.1)$ & $1285(21.0)$ & $0.94(0.88,1.01)$ & 0.0896 \\
\hline & Poorly Diff & $38441(55.0)$ & $7472(55.3)$ & $3748(61.3)$ & $1.04(0.97,1.10)$ & 0.2788 \\
\hline & Un Diff & 1195 ( 1.7$)$ & $151(1.1)$ & $121(2.0)$ & $1.04(0.89,1.21)$ & 0.6322 \\
\hline \multirow[t]{7}{*}{ Location n (\%) } & Cardia & $31016(44.3)$ & $1840(13.6)$ & 713 ( 11.7$)$ & Ref & \\
\hline & Fundus & $2474(3.5)$ & $473(3.5)$ & $171(2.8)$ & $0.78[0.68,0.90]$ & 0.0004 \\
\hline & Body & 4738 ( 6.8$)$ & $1240(9.2)$ & $581(9.5)$ & $1.26[1.16,1.37]$ & $<.0001$ \\
\hline & Antrum & 9528 ( 13.6$)$ & 3532 ( 26.2) & $1886(30.9)$ & $1.79[1.69,1.89]$ & $<.0001$ \\
\hline & Pylorus & $1368(2.0)$ & $525(3.9)$ & $216(3.5)$ & $1.42[1.25,1.61]$ & $<.0001$ \\
\hline & Overlapping & $10599(15.2)$ & 2937 ( 21.8$)$ & $1612(26.4)$ & $1.51[1.43,1.60]$ & $<.0001$ \\
\hline & NOS & 10222 ( 14.6$)$ & 2953 ( 21.9) & $934(15.3)$ & $0.95[0.88,1.01]$ & 0.1104 \\
\hline Year of Diagnosis n (\%) & 2004 & 7265 ( 10.4$)$ & 1267 ( 9.4$)$ & $622(10.2)$ & Ref & \\
\hline
\end{tabular}

J Surg Oncol. Author manuscript; available in PMC 2020 May 01. 


\begin{tabular}{|c|c|c|c|c|c|c|}
\hline & \multirow[b]{2}{*}{2005} & \multirow{2}{*}{$\begin{array}{c}\text { White } \\
6965(10.0)\end{array}$} & \multirow{2}{*}{$\begin{array}{c}\text { Black } \\
1323(9.8)\end{array}$} & \multirow{2}{*}{$\frac{\text { Asian }}{631(10.3)}$} & \multicolumn{2}{|c|}{ Multivariable OR Asian vs Others } \\
\hline & & & & & $0.98(0.91,1.06)$ & 0.6057 \\
\hline & 2006 & $7047(10.1)$ & $1377(10.2)$ & $596(9.7)$ & $0.94(0.87,1.02)$ & 0.1138 \\
\hline & 2007 & $7106(10.2)$ & $1456(10.8)$ & $657(10.7)$ & $1.00(0.93,1.08)$ & 0.9111 \\
\hline & 2008 & 7607 ( 10.9$)$ & $1538(11.4)$ & $603(9.9)$ & $0.88(0.81,0.95)$ & 0.0016 \\
\hline & 2009 & 7971 ( 11.4$)$ & $1579(11.7)$ & $745(12.2)$ & $1.08(1.00,1.16)$ & 0.0464 \\
\hline & 2010 & 8471 ( 12.1$)$ & $1606(11.9)$ & 711 ( 11.6$)$ & $1.02(0.94,1.09)$ & 0.6516 \\
\hline & 2011 & $8746(12.5)$ & $1644(12.2)$ & $753(12.3)$ & $1.07(1.00,1.15)$ & 0.0646 \\
\hline & 2012 & 8767 ( 12.5$)$ & $1710(12.7)$ & $795(13.0)$ & $1.12(1.04,1.20)$ & 0.0024 \\
\hline \multicolumn{7}{|l|}{ Treatment } \\
\hline \multirow[t]{3}{*}{ Surgery } & None & $34140(48.8)$ & $6416(47.5)$ & $2165(35.4)$ & Ref & \\
\hline & Ablation/EMR & $1643(2.3)$ & $167(1.2)$ & 87 ( 1.4$)$ & $0.88(0.75,1.02)$ & 0.0965 \\
\hline & Gastrectomy & $34162(48.8)$ & 6917 ( 51.2) & 3861 ( 63.2) & $1.26(1.16,1.37)$ & $<.0001$ \\
\hline \multirow{2}{*}{ Chemo } & None & $32230(46.1)$ & 6865 ( 50.9) & 3131 ( 51.2) & Ref & \\
\hline & chemo & 37715 ( 53.9) & $6635(49.1)$ & $2982(48.8)$ & $0.95(0.92,0.98)$ & 0.0008 \\
\hline \multirow{2}{*}{$R T$} & None & 48400 ( 69.2$)$ & $10055(74.5)$ & 4457 ( 72.9$)$ & Ref & \\
\hline & $\mathrm{RT}$ & 21545 ( 30.8$)$ & 3445 ( 25.5) & $1656(27.1)$ & $1.09(1.05,1.13)$ & $<.0001$ \\
\hline
\end{tabular}

Abbreviations: OR- odds ratio; CI- confidence interval; ref- reference; CDC- Charlson-Deyo comorbidity score; adeno- adenocarcinoma; SCCsquamous cell carcinoma; adenosquam- adenosquamous carcinoma; AJCC- American Joint Committee on Cancer; Well Diff- well differentiated; Mod Diff- moderately differentiated; Poorly Diff- poorly differentiated; Un Diff- undifferentiated; NOS- not otherwise specified; EMRendomucosal resection; chemo- chemotherapy; RT- radiation therapy 
Table 2.

Predictors of overall survival in entire cohort and Asian subgroup with interaction set between stage and treatment type.

Multivariable HR estimated from the full Cox proportional hazards model including all covariates in this table and interaction between Stage and treatment modality combination.

\begin{tabular}{|c|c|c|c|c|}
\hline & \multicolumn{2}{|l|}{ Whole Cohort $(\mathrm{N}=\mathbf{8 9 , 5 5 8})$} & \multicolumn{2}{|l|}{ Asians only $(\mathrm{N}=6,113)$} \\
\hline & Multivariable Hazard Ratio [95\% CI] & P-value & Multivariable Hazard Ratio [95\% CI] & P-value \\
\hline \multicolumn{5}{|l|}{ Race } \\
\hline White & Ref & & - & - \\
\hline Black & $0.98[0.95,1.00]$ & 0.035 & - & - \\
\hline \multicolumn{5}{|l|}{ Asian } \\
\hline Chinese & $0.69[0.64,0.75]$ & $<.0001$ & Ref & \\
\hline Korean & $0.70[0.64,0.75]$ & $<.0001$ & $0.99[0.89,1.11]$ & 0.8936 \\
\hline Japanese & $0.84[0.77,0.91]$ & $<.0001$ & $1.21[1.07,1.36]$ & 0.0017 \\
\hline Vietnamese & $0.79[0.71,0.88]$ & $<.0001$ & $1.11[0.97,1.26]$ & 0.1327 \\
\hline Indian & $0.68[0.61,0.76]$ & $<.0001$ & $0.93[0.81,1.08]$ & 0.3427 \\
\hline Filipino & $1.00[0.89,1.12]$ & 0.9596 & $1.44[1.25,1.65]$ & $<.0001$ \\
\hline Other & $0.69[0.64,0.75]$ & $<.0001$ & $0.97[0.87,1.08]$ & 0.5401 \\
\hline Age over 60 & $1.17[1.15,1.20]$ & $<.0001$ & $1.16[1.06,1.26]$ & 0.0008 \\
\hline \multicolumn{5}{|l|}{ Gender } \\
\hline Female & Ref & & Ref & \\
\hline Male & $1.03[1.02,1.05]$ & 0.0002 & $0.92[0.86,0.99]$ & 0.0176 \\
\hline \multicolumn{5}{|l|}{ Facility Type } \\
\hline Non-Academic & Ref & & Ref & \\
\hline Academic/Research Program & $0.84[0.82,0.85]$ & $<.0001$ & $0.83[0.78,0.89]$ & $<.0001$ \\
\hline \multicolumn{5}{|l|}{ Insurance Type } \\
\hline Uninsured & Ref & & Ref & \\
\hline Private & $1.00[0.96,1.04]$ & 0.9075 & $0.92[0.79,1.07]$ & 0.2743 \\
\hline Government & $1.16[1.11,1.21]$ & $<.0001$ & $1.13[0.97,1.32]$ & 0.1148 \\
\hline \multicolumn{5}{|l|}{ CDCC Total } \\
\hline 0 & Ref & & Ref & \\
\hline 1 & $1.14[1.12,1.16]$ & $<.0001$ & $1.10[1.01,1.20]$ & 0.0345 \\
\hline 2 & $1.37[1.33,1.41]$ & $<.0001$ & $1.20[1.04,1.39]$ & 0.0157 \\
\hline \multicolumn{5}{|l|}{ Histology } \\
\hline Adenocarcinoma & Ref & & Ref & \\
\hline SCC & $1.24[1.09,1.41]$ & 0.0008 & $0.94[0.48,1.84]$ & 0.8655 \\
\hline Adenosquamous & $1.13[1.05,1.22]$ & 0.0011 & $1.35[0.80,2.27]$ & 0.2593 \\
\hline
\end{tabular}




\begin{tabular}{|c|c|c|c|c|}
\hline & \multicolumn{2}{|l|}{ Whole Cohort $(\mathrm{N}=\mathbf{8 9 , 5 5 8})$} & \multicolumn{2}{|l|}{ Asians only $(\mathrm{N}=6,113)$} \\
\hline & Multivariable Hazard Ratio [95\% CI] & P-value & Multivariable Hazard Ratio [95\% CI] & P-value \\
\hline Well Differentiated & Ref & & Ref & \\
\hline Mod Differentiated & $1.02[0.87,1.20]$ & 0.8188 & $1.13[0.90,1.42]$ & 0.2856 \\
\hline Poorly Differentiated & $1.24[1.07,1.44]$ & 0.0057 & $1.33[1.09,1.64]$ & 0.0064 \\
\hline Undifferentiated & $1.34[1.15,1.56]$ & 0.0003 & $1.69[1.19,2.41]$ & 0.0038 \\
\hline \multicolumn{5}{|l|}{ Location } \\
\hline Cardia & Ref & & Ref & \\
\hline Fundus & $1.12[1.07,1.17]$ & $<.0001$ & $1.05[0.84,1.31]$ & 0.6744 \\
\hline Body & $1.01[0.98,1.05]$ & 0.5568 & $1.06[0.91,1.23]$ & 0.4827 \\
\hline Antrum & $1.01[0.98,1.04]$ & 0.4253 & $1.03[0.91,1.16]$ & 0.6552 \\
\hline Pylorus & $1.01[0.96,1.06]$ & 0.7548 & $1.03[0.91,1.16]$ & 0.6552 \\
\hline Overlapping & $1.05[1.02,1.07]$ & 0.0004 & $1.01[0.89,1.14]$ & 0.9333 \\
\hline NOS & $1.13[1.10,1.16]$ & $<.0001$ & $1.15[1.01,1.31]$ & 0.0304 \\
\hline \multicolumn{5}{|l|}{ Year of Diagnosis } \\
\hline 2004 & Ref & & Ref & \\
\hline 2005 & $0.98[0.95,1.01]$ & 0.2286 & $1.02[0.89,1.16]$ & 0.8179 \\
\hline 2006 & $0.95[0.92,0.98]$ & 0.0023 & $0.92[0.79,1.06]$ & 0.2226 \\
\hline 2007 & $0.96[0.93,0.99]$ & 0.0111 & $0.94[0.81,1.08]$ & 0.3544 \\
\hline 2008 & $0.96[0.93,0.99]$ & 0.0186 & $0.91[0.79,1.05]$ & 0.2183 \\
\hline 2009 & $0.93[0.90,0.96]$ & $<.0001$ & $0.91[0.79,1.05]$ & 0.1845 \\
\hline 2010 & $0.92[0.89,0.95]$ & $<.0001$ & $0.94[0.81,1.08]$ & 0.3712 \\
\hline 2011 & $0.85[0.82,0.88]$ & $<.0001$ & $0.90[0.78,1.04]$ & 0.1623 \\
\hline 2012 & $0.84[0.81,0.87]$ & $<.0001$ & $0.77[0.66,0.90]$ & 0.0009 \\
\hline \multicolumn{5}{|l|}{ Stage $\&$ Treatment } \\
\hline \multicolumn{5}{|l|}{ Stage 1} \\
\hline No treatment & Ref & & Ref & \\
\hline Surgery only & $0.20[0.17,0.23]$ & $<.0001$ & $0.19[0.15,0.24]$ & $<.0001$ \\
\hline Chemo only & $0.86[0.76,0.96]$ & 0.0105 & $1.14[0.70,1.85]$ & 0.6047 \\
\hline RT only & $0.97[0.82,1.15]$ & 0.7021 & $1.77[1.02,3.06]$ & 0.0411 \\
\hline Chemo+RT & $0.59[0.52,0.67]$ & $<.0001$ & $0.68[0.36,1.31]$ & 0.2490 \\
\hline Surgery+Chemo & $0.18[0.15,0.22]$ & $<.0001$ & $0.20[0.12,0.32]$ & $<.0001$ \\
\hline Surgery+RT & $0.22[0.17,0.30]$ & $<.0001$ & $0.34[0.13,0.87]$ & 0.0237 \\
\hline Surgery+ChemoRT & $0.22[0.19,0.26]$ & $<.0001$ & $0.15[0.10,0.22]$ & $<.0001$ \\
\hline \multicolumn{5}{|l|}{ Stage 2} \\
\hline No treatment & Ref & & Ref & \\
\hline Surgery only & $0.34[0.28,0.40]$ & $<.0001$ & $0.41[0.28,0.60]$ & $<.0001$ \\
\hline Chemo only & $0.62[0.54,0.71]$ & $<.0001$ & $0.95[0.54,1.65]$ & 0.8466 \\
\hline RT only & $0.88[0.74,1.04]$ & 0.1287 & $1.31[0.45,3.76]$ & 0.6187 \\
\hline Chemo+RT & $0.45[0.40,0.52]$ & $<.0001$ & $0.64[0.35,1.16]$ & 0.1445 \\
\hline Surgery+Chemo & $0.22[0.18,0.26]$ & $<.0001$ & $0.25[0.16,0.39]$ & $<.0001$ \\
\hline
\end{tabular}




\begin{tabular}{|c|c|c|c|c|}
\hline & \multicolumn{2}{|l|}{ Whole Cohort $(\mathrm{N}=\mathbf{8 9 , 5 5 8})$} & \multicolumn{2}{|l|}{ Asians only $(\mathrm{N}=6,113)$} \\
\hline & Multivariable Hazard Ratio [95\% CI] & P-value & Multivariable Hazard Ratio [95\% CI] & P-value \\
\hline Surgery+RT & $0.21[0.16,0.27]$ & $<.0001$ & $0.39[0.19,0.77]$ & 0.0067 \\
\hline Surgery+ChemoRT & $0.20[0.16,0.23]$ & $<.0001$ & $0.21[0.15,0.32]$ & $<.0001$ \\
\hline \multicolumn{5}{|l|}{ Stage 3} \\
\hline No treatment & Ref & & Ref & \\
\hline Surgery only & $0.48[0.43,0.54]$ & $<.0001$ & $0.50[0.36,0.70]$ & $<.0001$ \\
\hline Chemo only & $0.56[0.51,0.61]$ & $<.0001$ & $0.89[0.59,1.36]$ & 0.6042 \\
\hline RT only & $0.74[0.64,0.87]$ & 0.0002 & $1.10[0.61,1.98]$ & 0.7546 \\
\hline Chemo+RT & $0.46[0.42,0.50]$ & $<.0001$ & $0.71[0.46,1.09]$ & 0.1196 \\
\hline Surgery + Chemo & $0.30[0.26,0.33]$ & $<.0001$ & $0.39[0.27,0.55]$ & $<.0001$ \\
\hline Surgery+RT & $0.33[0.27,0.39]$ & $<.0001$ & $0.27[0.14,0.52]$ & $<.0001$ \\
\hline Surgery+ChemoRT & $0.25[0.23,0.28]$ & $<.0001$ & $0.29[0.21,0.40]$ & $<.0001$ \\
\hline \multicolumn{5}{|l|}{ Stage 4} \\
\hline No treatment & Ref & & Ref & \\
\hline Surgery only & $0.42[0.40,0.44]$ & $<.0001$ & $0.47[0.40,0.56]$ & $<.0001$ \\
\hline Chemo only & $0.37[0.36,0.38]$ & $<.0001$ & $0.41[0.36,0.46]$ & $<.0001$ \\
\hline RT only & $0.77[0.73,0.82]$ & $<.0001$ & $0.75[0.58,0.98]$ & 0.0318 \\
\hline Chemo+RT & $0.35[0.33,0.36]$ & $<.0001$ & $0.39[0.32,0.48]$ & $<.0001$ \\
\hline Surgery + Chemo & $0.23[0.22,0.24]$ & $<.0001$ & $0.26[0.21,0.31]$ & $<.0001$ \\
\hline Surgery+RT & $0.28[0.23,0.34]$ & $<.0001$ & $0.23[0.12,0.45]$ & $<.0001$ \\
\hline Surgery+ChemoRT & $0.17[0.16,0.18]$ & $<.0001$ & $0.17[0.13,0.20]$ & $<.0001$ \\
\hline
\end{tabular}

Abbreviations: CI- confidence interval; ref- reference; CDC- Charlson-Deyo comorbidity score; SCC- squamous cell carcinoma; AJCC- American Joint Committee on Cancer; NOS- not otherwise specified; chemo- chemotherapy; RT- radiation therapy 